\title{
Intercalant and intermolecular phonon assisted superconductivity in K-doped picene
}

\author{
Michele Casula, ${ }^{1}$ Matteo Calandra, ${ }^{1}$ Gianni Profeta ${ }^{2}$ and Francesco Mauri ${ }^{1}$ \\ 1 IMPMC, Université Paris 6, CNRS, 4 Place Jussieu, 7504 Paris, France \\ ${ }^{2}$ SPIN-CNR - Dip. di Fisica Università degli Studi di L'Aquila, 67100 L'Aquila, Italy \\ and Max-Planck Institute of Microstructure Physics, Weinberg 2, 06120 Halle, Germany
}

(Dated: March 6, 2018)

\begin{abstract}
$\mathrm{K}_{3}$-picene is a superconducting molecular crystal with critical temperature $\mathrm{T}_{c}=7 \mathrm{~K}$ or $18 \mathrm{~K}$, depending on preparation conditions. Using density functional theory we show that electron-phonon interaction accounts for $T_{c} 3-8 \mathrm{~K}$. The average electron-phonon coupling, calculated by including the phonon energy scale in the electron-phonon scattering, is $\lambda=0.73$ and $\omega_{\log }=18.0 \mathrm{meV}$. Intercalant and intermolecular phonon-modes contribute substantially (40\%) to $\lambda$ as also shown by the isotope exponents of potassium (0.19) and carbon (0.31). The relevance of these modes makes superconductivity in K-doped picene peculiar and different from that of fullerenes.

PACS numbers: 31.15.A-, 74.70.Kn, 63.20.kd
\end{abstract}

Most materials made of organic molecules are not metals as the valence bands are completely filled and separated by the conduction band. However, the discovery in 1980 of superconducting tetra-methyl-tetraselenium-fulvalene [1], demonstrated the possibility to induce superconductivity by intercalation of large organic molecules with inorganic anions, serving as acceptors or donors. This general rule was confirmed by the discovery of superconductivity in alkali-doped fullerenes [2] with transition temperatures $\left(T_{c}\right)$ up to $40 \mathrm{~K}^{\text {in }} \mathrm{Cs}_{3} \mathrm{C}_{60}[3]$.

The field was renewed recently with the discovery of superconductivity in potassium $(\mathrm{K})$-intercalated molecularsolids based on polycyclic aromatic hydrocarbons, such as picene [4] $\left(\mathrm{T}_{c}=7 \mathrm{~K}\right.$ or $\left.\mathrm{T}_{c}=18 \mathrm{~K}[5]\right)$, phenantrene [6] $\left(\mathrm{T}_{c}=5 \mathrm{~K}\right)$ and coronene[7] $\left(\mathrm{T}_{c}=15 \mathrm{~K}\right)$. These systems are very appealing as they exist in a large variety, and it is possible to tune their properties in many ways. For instance, the chemistry and functionalization of polycyclic hydrocarbons is well known and relevant for environmental and medical issues [8], the intercalation with alkali atoms is often possible, and the electrochemical doping in picene field-effect transistors has been achieved [9].

In addition, organic molecular crystals are particularly appealing for technological applications such as organic light emitting diodes (OLED), field effect transistors (OFET) or solar cells. Consequently, the study of charge injection and carrier transport in these materials are central to the understanding and improvement of such devices. In particular, the study of the electronphonon coupling interaction is crucial to describe the different regimes of charge transport [10].

In molecular crystals, the electrons can couple to three kinds of vibrations; intramolecular, intermolecular (rigid translations and rotations of molecules) and intercalant phonons. In alkali-doped $\mathrm{C}_{60}$, after a long debate, 2, 1114], it has been established that superconductivity is driven mainly by intramolecular modes. This conclusion is also supported by the negligible $\mathrm{Rb}$ isotope effect measured in $\mathrm{Rb}_{3} \mathrm{C}_{60}[15,16]$. However the situation could be substantially different in K-doped Picene where a more prominent role of intercalant modes in superconductivity is suggested by the significant hybridization of the lowenergy bands with $\mathrm{K}$ atoms observed in density functional theory (DFT) [17]. Indeed, the electronic structure of K-doped picene is different from that obtained by a rigid-doping of the picene crystal without explicit $\mathrm{K}$ ions [17. Thus, at the moment, it is still unclear (i) if superconductivity in K-doped picene is phonon-mediated, (ii) if the intramolecular phonons alone provide enough coupling or if intercalant and intermolecular vibrations should be also considered.

These questions were first addressed in an early DFT calculation [18] where it was found a sizable electronphonon coupling, dominated by high-energy intramolecular in-plane carbon $(\mathrm{C})$ vibrations at $1000 \mathrm{~cm}^{-1}$. However, this work uses a rigid-band doping approximation of the pristine picene crystal structure and thus neglects the molecular relaxation after intercalation, the $\mathrm{K}$ contribution to the electron-phonon coupling, and the screening of the electron-phonon deformation-potential by the metallic bands. Furthermore only modes above $100 \mathrm{~cm}^{-1}$ were considered.

In this letter, we perform first-principles DFT calculations on the superconducting properties of $\mathrm{K}_{3}$-picene. Removing all possible a-priori approximations, and in contrast to [18], we demonstrate the critical role played by the intercalant and intermolecular modes in the electron-phonon coupling mechanism to account for the experimental $T_{c}$. We give a numerical prediction of the isotope exponents in the molecular crystal and relate them to the phonon-mediated superconductivity.

We compute the electronic structure of $\mathrm{K}_{3}$-picene within DFT in the local density approximation (LDA) [19]. We relax the molecular geometry in the $\mathrm{P} 2_{1}$ symmetry in the experimental unit cell [4, 21], obtaining a structure similar to that of [17] with the molecules arranged in a herringbone configuration and the intercalants aligned parallel to the molecular planes 22 . 


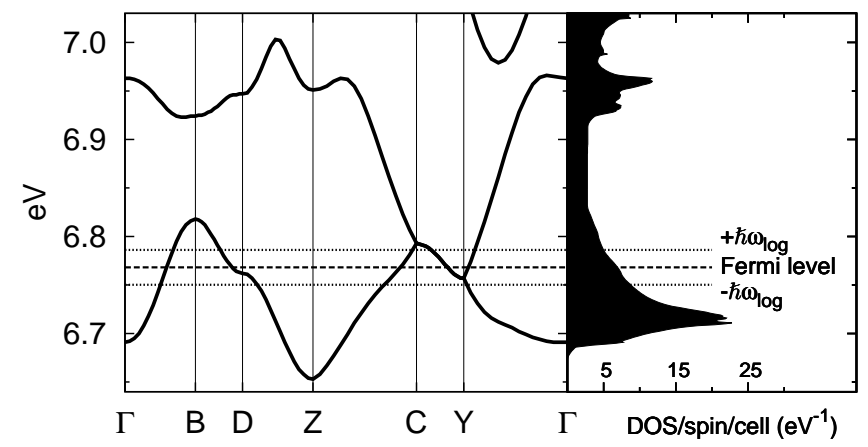

FIG. 1: DFT-LDA band structure of $\mathrm{K}_{3}$-picene near the Fermi level. The DOS per spin per cell (comprising 2 picene molecules) is shown in the right panel. Note the strong DOS variation in a range of $\pm \hbar \omega_{l o g}$ from the Fermi energy, with the characteristic phonon frequency $\omega_{l o g}=18 \mathrm{meV}$

The band structure of the $K_{3}$ picene is reported in Fig. 1. The molecular nature of the compound gives rise to very narrow bands $\epsilon_{\mathbf{k} n}$, with bandwidth $\Delta \epsilon$ of few tenths of $\mathrm{eV}$. The Fermi level $\epsilon_{F}$ in the K-doped picene crosses the bands originating from the LUMO+1 (second Lowest Unoccupied Molecular Orbital) states of the undoped molecule, which are entangled to those coming from the LUMO +2 states, and very close to the ones belonging to the LUMO bands. The bandwidth $\Delta \epsilon$ has the same order of magnitude as the characteristic phonon frequency $\omega_{p h}$ [the logarithmic phonon average $\omega_{l o g}=18 \mathrm{meV}$, calculated in this work (see below)]. The density of states (DOS) varies substantially in the range $\left[\epsilon_{F}-\omega_{l o g}, \epsilon_{F}+\omega_{l o g}\right]$, as shown in Fig. [1.

We carry out phonon calculations in the density functional perturbation theory framework 24]. We consider phonon momenta on a $N_{q}=2 \times 2 \times 2$ grid. We get dynamically stable phonons, signaling a well converged phonon dispersion $\omega_{\mathbf{q} \nu}$ and no structural instabilities in the chosen geometry. In Fig2(c) we report the projected phonon DOS, $\rho_{S}(\epsilon)=\sum_{\mathbf{q} \nu} \mathbf{e}_{\mathbf{q} \nu}^{*} \cdot \mathcal{P}_{S} \mathbf{e}_{\mathbf{q} \nu} \delta\left(\omega_{\mathbf{q} \nu}-\epsilon\right)$, where the phonon eigenstates $\mathbf{e}_{\mathbf{q} \nu}$ are $3 \mathrm{~N}$-dimensional vectors, with $\mathrm{N}$ the number of atoms, and the projector $\mathcal{P}_{S}$ is a $3 \mathrm{~N} \times 3 \mathrm{~N}$ tensor. We split the full phonon space into potassium $(\mathrm{K})$, hydrogen $(\mathrm{H})$, in-plane $\left(\mathrm{C}_{=}\right)$and out-of-plane $\mathrm{C}_{\perp}$ carbon $S$-subspaces (the plane is the one containing each picene molecule). We also consider partitioning into $\mathrm{K}$, intermolecular and intramolecular vibrations. The projections reveal the presence of the $\mathrm{K}$ and intermolecular modes at low frequencies, while only a very small fraction of intramolecular modes is present below $200 \mathrm{~cm}^{-1}$. On the other hand, the in-plane $\mathrm{C}$ and $\mathrm{H}$ modes occupy the high-energy part of the DOS. We compute the phonon spectrum also for the neutral isolated molecule, Fig2(d). The low-energy spectrum of molecule and crystal differ considerably. In the solid the rigidbody rototranslations (intermolecular modes) are spread up to $300 \mathrm{~cm}^{-1}$, while in the molecule they are pinned at zero frequency. In addition we observe in the crystal a significant spectral weight transfer of the low-energy out-of-plane $\mathrm{C}$ oscillations to higher frequencies.

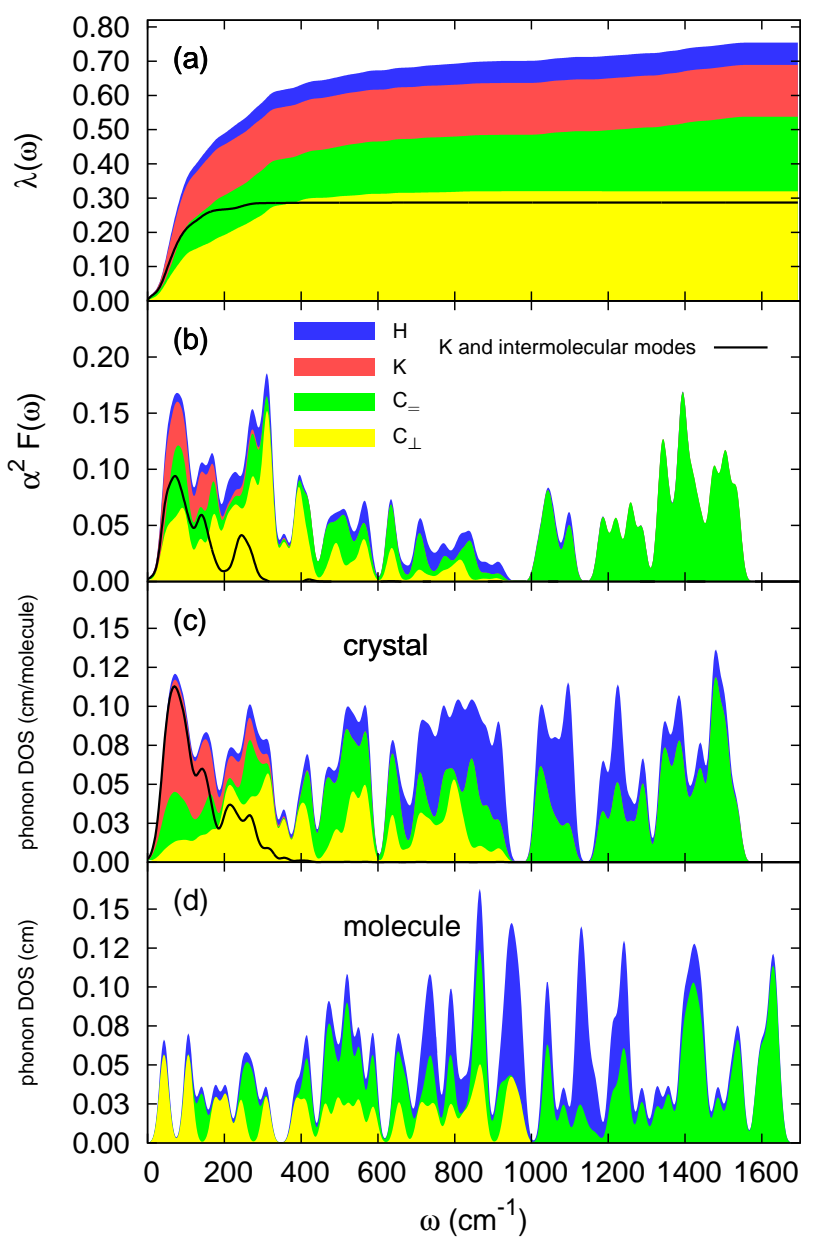

FIG. 2: Panels (c) and (d): phonon DOS of the $\mathrm{K}_{3}$-picene crystal and picene molecule, respectively. The DOS is projected on phonon subspaces $S$ which correspond to hydrogen $(\mathrm{H})$, potassium $(\mathrm{K})$, out-of-plane and in-plane carbon $\left(\mathrm{C}_{\perp}\right.$ and $\mathrm{C}_{=}$) modes. The black solid curve is the sum of $\mathrm{K}$ and intermolecular modes. In the (d) panel the black line is absent as there is no $\mathrm{K}$ and all phonons are intramolecular, except for the six rototranslational modes at zero frequency. We do not show the $\mathrm{C}-\mathrm{H}$ stretching modes, which do not couple to electrons, and are located around $3050 \mathrm{~cm}^{-1}$, well above the frequency range plotted. Panels (a) and (b): $\lambda(\omega)$ and $\alpha^{2} F(\omega)$ resolved by projections on $\mathrm{H}, \mathrm{K}, \mathrm{C}_{\perp}$, and $\mathrm{C}_{=}$phonons. $\lambda(\omega)$ ramps up by $90 \%$ in the first $300 \mathrm{~cm}^{-1}$. The black solid line is the sum of $\mathrm{K}$ and intermolecular modes, which contribute to $40 \%$ of the total $\lambda$.

For each phonon mode $\nu$ with momentum q we compute the electron-phonon interaction:

$$
\begin{aligned}
\lambda_{\mathbf{q} \nu} & =\frac{2}{\omega_{\mathbf{q} \nu}^{2} N(0) N_{k}} \sum_{\mathbf{k}, n, m}\left|g_{\mathbf{k} n, \mathbf{k}+\mathbf{q} m}^{\nu}\right|^{2} \\
& \times\left(f_{\mathbf{k} n}-f_{\mathbf{k}+\mathbf{q}, m}\right) \delta\left(\epsilon_{\mathbf{k}+\mathbf{q}, m}-\epsilon_{\mathbf{k} n}-\omega_{\mathbf{q} \nu}\right),
\end{aligned}
$$


that couples the occupied state $|\mathbf{k}, n\rangle$ of momentum $\mathbf{k}$ and band $n$ with the empty state $|\mathbf{k}+\mathbf{q}, m\rangle$ separated by the phonon energy $\omega_{\mathbf{q} \nu}$. The $\mathbf{k}$-summation in Eq. 1 is over the Brillouin zone on a $N_{k}$ electron-momentum mesh, and $N(0)\left(=6.2 \mathrm{eV}^{-1}\right)$ is the electron DOS per spin per cell at the Fermi level. The electron-phonon matrix elements are $g_{\mathbf{k} n, \mathbf{k}+\mathbf{q} m}^{\nu}=\sum_{s} \mathbf{e}_{\mathbf{q} \nu}^{s} \cdot \mathbf{d}_{n m}^{s}(\mathbf{k}, \mathbf{k}+\mathbf{q}) / \sqrt{2 M_{s} \omega_{\mathbf{q} \nu}}$, where $\mathbf{e}_{\mathbf{q} \nu}^{s}$ is the 3 -dimensional $\mathbf{q} \nu$ eigenphonon component on the $s$-th atom with mass $M_{s}$, and $\mathbf{d}_{n m}^{s}(\mathbf{k}, \mathbf{k}+\mathbf{q})=$ $\left\langle\mathbf{k}+\mathbf{q}, m\left|\delta V / \delta u_{\mathbf{q} s}\right| \mathbf{k}, n\right\rangle$, with $\delta V / \delta u_{\mathbf{q} s}$ the deformation potential for a given phonon displacement $\mathbf{q} s$ of the $s$ th atom. In Eq. 1, $f_{\mathbf{k} n}$ are Fermi functions depending on the temperature $T$, and the expression for $\lambda_{\mathbf{q} \nu}$ has been evaluated by a $T \rightarrow 0$ extrapolation. In the "adiabatic" limit, namely for $\omega_{p h} \ll \Delta \epsilon$, where $\Delta \epsilon$ is the bandwith, the expression for $\lambda_{\mathrm{q} \nu}$ in Eq. 11 reduces to the one proposed by Allen [25], and generally used in previous electron-phonon estimates:

$$
\lambda_{\mathbf{q} \nu}^{A D}=\frac{2}{\omega_{\mathbf{q} \nu} N(0) N_{k}} \sum_{\mathbf{k}, n, m}\left|g_{\mathbf{k} n, \mathbf{k}+\mathbf{q} m}^{\nu}\right|^{2} \delta\left(\epsilon_{\mathbf{k}, n}\right) \delta\left(\epsilon_{\mathbf{k}+\mathbf{q}, m}\right) .
$$

We are going to dub $\lambda^{A D}$ in the Equation above as "adiabatic", while $\lambda$ in Eq. 1 as "non-adiabatic".

The electron-phonon matrix elements $g_{\mathbf{k} n, \mathbf{k}+\mathbf{q} m}^{\nu}$ are computed on a highly dense electron-momentum grid by means of the recently developed Wannier interpolation formalism [26 30]. This allows one to perform converged calculations with a delta function Gaussian smearing $\sigma$ much smaller than the bandwidth $\Delta \epsilon$ [31]. Surprisingly, the exact zero-smearing extrapolation leads to a strong electron and phonon-momentum dependence in $g$, and so in $\lambda$ and $\lambda^{A D}$. This is in contrast with the conventional molecular crystal picture, where $g$ and the electron-phonon coupling are independent of the electron and phonon momenta. To quantify this dependence, we evaluated the coupling strength average per molecule, given by $2 \lambda_{\mathbf{q}}^{A D} N(0) / J_{\mathbf{q}}$, where $J_{\mathbf{q}}=$ $\sum_{\mathbf{k}, n, m} \delta\left(\epsilon_{\mathbf{k}, n}\right) \delta\left(\epsilon_{\mathbf{k}+\mathbf{q}, m}\right) / N_{k}$ is the nesting factor, $\lambda_{\mathbf{q}}^{A D}$ is summed over the phonon modes, and the factor of 2 accounts for the number of molecules in the unit cell. One obtains the phonon-momentum integrated average-value of $172 \mathrm{meV}$, with large variations of $\pm 40 \%$ as a function of the phonon-momentum 23. A detailed analysis of the dependence of $\lambda$ on $\mathbf{q}$ and $\sigma$ is presented in [23].

In Tab. [. we report our results for the electron-phonon coupling. There is a significant difference between the "adiabatic" and "non-adiabatic" values, as $\omega_{p h} \sim \Delta \epsilon$. Therefore, one must keep the full expression in Eq. 1 for an accurate estimate of $\lambda$. In Tab. If we also report the phonon frequency logarithmic average $\left(\omega_{\text {log }}\right)$.

An estimate of $T_{c}$ is given by the McMillan formula [32], which requires $\lambda, \omega_{l o g}$, and the screened Coulomb pseudopotential $\mu^{*}$. With $\mu^{*} \in[0.1,0.2]$, we obtain $T_{c}$ in the range $3-8 \mathrm{~K}$, which includes the value of $7 \mathrm{~K}$ reported in [4] for one of the two phases of $\mathrm{K}_{3^{-}}$

\begin{tabular}{|l|c|c|c|c|c|c|}
\hline & $\lambda$ & $\omega_{\log }(\mathrm{meV})$ & $\begin{array}{c}T_{c}(\mathrm{~K}) \\
\mu^{*} \in[0.1,0.2]\end{array}$ & $\alpha(\mathrm{C})$ & $\alpha(\mathrm{K})$ & $\alpha(\mathrm{H})$ \\
\hline adiabatic & 0.88 & 25 & $8-16$ & 0.32 & 0.13 & 0.05 \\
\hline non-adiabatic & 0.73 & 18 & $3-8$ & 0.31 & 0.19 & 0.00 \\
\hline
\end{tabular}

TABLE I: $\lambda$, phonon frequency logarithmic average $\omega_{\text {log }}$, McMillan critical temperature $T_{c}$ (obtained with $\mu^{*}$ in the range of 0.1-0.2), and isotope exponents (for $\mu^{*}=0.14$ ), computed based on the electron-phonon coupling expression in Eq. 1 (non-adiabatic), which explicitly includes the phonon energy $\omega$ in the electron-phonon scattering process, and the one in Eq. 2 (adiabatic), which is a $\omega_{p h} / \Delta \epsilon \rightarrow 0$ approximation of Eq. 1] The "non-adiabatic" values are our best estimates of the various quantities.

picene [5]. Caution must be taken in using the McMillan formula, as the on-site molecular correlation can be important with respect to the bandwidth [18, 33], and also "non-adiabatic" effects in the vertex corrections can invalidate the Migdal's theorem 34]. However, our estimate of $T_{c}$ is an indication that the electron-phonon coupling is strong enough to explain the mechanism behind the manifestation of the superconducting phase. We computed also the isotope exponents $\alpha(X)=-d \log T_{c} / d \log M_{x}$ (see Tab. I) for all constituents of the molecular crystal. It turns out that the role of $\mathrm{H}$ is negligible, while the large exponent of potassium $(\mathrm{K})$ points toward the important role played by the intercalant in the electronphonon coupling to set the value of $T_{c}$.

For a given value of $\mu^{*}$ our predicted $T_{c}$ are more than 8 times smaller than that predicted with $\lambda$ and $\omega_{\text {log }}$ found in [18]. This comes mainly from the value of $\omega_{\log }$ (1021 $\mathrm{cm}^{-1}$ ) in [18], very different from our best estimate of 18 $\mathrm{meV}\left(145 \mathrm{~cm}^{-1}\right)$. This large discrepancy may come from the intercalation driven structural change not considered in that work. Indeed, the electronic DOS of $\mathrm{K}_{3}$-picene, computed in the rigid-doping approximation with the undoped geometry, is $14.12 \mathrm{eV}^{-1}$ per spin per cell [17, 18], a value 2.3 larger than that found here and in [17] for the relaxed geometry with K. In addition, [18] neglects the screening of the electron-phonon interaction by the metallic bands, the contribution of $\mathrm{K}$ phonons and vibrations of energy lower than $100 \mathrm{~cm}^{-1}$.

To understand our findings, we compute the Eliashberg function $\alpha^{2} F(\omega)=\sum_{\mathbf{q} \nu} \lambda_{\mathbf{q} \nu} \omega_{\mathbf{q} \nu} \delta\left(\omega-\omega_{\mathbf{q} \nu}\right) /\left(2 N_{q}\right)$, plotted in Fig. 2(b), and the integral $\lambda(\omega)=$ $2 \int_{0}^{\omega} d \omega^{\prime} \alpha^{2} F\left(\omega^{\prime}\right) / \omega^{\prime}$, shown in Fig. 2(a), which gives the total electron phonon coupling $\lambda=\sum_{\mathbf{q} \nu} \lambda_{\mathbf{q} \nu} / N_{q}$ in the $\omega \rightarrow \infty$ limit. We note that $\lambda(\omega)$ converges very rapidly to a large fraction of the total value in the first $300 \mathrm{~cm}^{-1}$.

We then decompose $\lambda$ into a set of phonon subspaces $S$, as we did for the phonon DOS, as in [35]. The evaluation of the projected electron-phonon matrix elements $g_{S}=\sum_{s}\left(\mathcal{P}_{S} \mathbf{e}_{\mathbf{q} \nu}\right)^{s} \cdot \mathbf{d}_{n m}^{s}(\mathbf{k}, \mathbf{k}+\mathbf{q}) / \sqrt{2 M_{s} \omega_{\mathbf{q} \nu}}$ follows straightforwardly, and the corresponding projected $\lambda_{\mathbf{q} \nu}^{S, S^{\prime}}$ 
reads:

$$
\begin{aligned}
\lambda_{\mathbf{q} \nu}^{S, S^{\prime}} & =\frac{2}{\omega_{\mathbf{q} \nu}^{2} N(0) N_{k}} \sum_{\mathbf{k}, n, m} g_{S} g_{S^{\prime}}^{\star} \\
& \times\left(f_{\mathbf{k} n}-f_{\mathbf{k}+\mathbf{q}, m}\right) \delta\left(\epsilon_{\mathbf{k}+\mathbf{q}, m}-\epsilon_{\mathbf{k} n}-\omega_{\mathbf{q} \nu}\right),
\end{aligned}
$$

where for the sake of readability we dropped out the momentum and orbital indexes from $g_{S} \cdot \lambda=\sum_{S, S^{\prime}} \lambda^{S, S^{\prime}}=$ $\sum_{S, S^{\prime}} \sum_{\mathbf{q}, \nu} \lambda_{\mathbf{q} \nu}^{S, S^{\prime}}$, and the contribution of each subspace $S$ is computed as $\sum_{S^{\prime}} \lambda^{S, S^{\prime}}$, where we add both the diagonal term and the usually very small off-diagonal contributions. The sum over $\mathrm{K}$ and intermolecular modes is reported in Fig. 2 as a black solid line. It gives more than $40 \%$ of the total $\lambda$, with half of it due to the alkali intercalant, and the corresponding $\alpha^{2} F(\omega)$ localized at small frequencies (up to $300 \mathrm{~cm}^{-1}$ ). The intramolecular phonons contribute to the remaining $60 \%$, with the main role played by the out-of-plane $\mathrm{C}$ modes. This is a remarkable result, and shows that $\mathrm{K}$-doped picene is far from being a prototype molecular crystal. The electron-phonon coupling is only partially supported by intramolecular phonons, and the q-dependence of the electron-phonon coupling is strong.

To summarize, we found that in the $\mathrm{K}_{3}$-picene the electron-phonon coupling is large enough to explain the experimentally measured $T_{c}$ of $7 \mathrm{~K}$. Despite the molecular nature of the crystal, a significant contribution to the coupling is given by the dopant and intermolecular phonon modes, which account for $40 \%$ of the total $\lambda$. This has a strong impact on the isotope exponent of potassium, whose value turns out to be large (close to 0.20 ), and represents an experimentally-accessible signature of the importance of non-intramolecular modes. Our work shows that there are fundamental differences between the families of picene and fullerene. The demonstration of the importance of coupling between the electrons at the Fermi level with intermolecular and intercalant low-energy phonon modes will have also a relevant contribution to the developing of transport theories, usually accounting for the local electron-phonon coupling (intramolecular coupling) and considered in a small-polaron theory of narrow bands. Extensions to include non-local couplings (intermolecular coupling) are required [36]. This opens the way to a more comprehensive view on superconductivity, transport, and other phenomena in the larger and larger group of recently discovered materials based on metal-intercalated aromatic hydrocarbons.

We acknowledge Y. Kubozono for useful discussions. Computer allocation has been supported by CINECAHPC ISCRA and EU DEISA-SUPERMAG.

[1] D. Jèrome et al., J. Phys. (Paris), Lett. 41 L95 (1980).
[2] O. Gunnarsson, Rev. Mod. Phys. 69, 575 (1997).

[3] T. T. M. Palstra et al.. Sol. State Comm. 93, 327 (1995).

[4] R. Mitsuhashi et al. Nature 464, 76 (2010).

[5] The observed $7 \mathrm{~K}$ and $18 \mathrm{~K} \mathrm{~T} \mathrm{~T}_{c}$ [4] likely correspond to 2 distinct crystalline phases of K-doped picene, Y. Kubozono, private communication.

[6] X. F. Wang et al., arXiv:1102.4075

[7] Y. Kubozono et al., submitted.

[8] Hydrocarbons chemistry, G. A. Olah and A. Molnar, Wiley Interscience 2003.

[9] H. Okamoto et al. J. Am. Chem. Soc. 130, 10470 (2008).

[10] F. Ortmann, F. Bechstedt and K. Hannewald, Phys. Status Solidi B 248, 511 (2011).

[11] F. C. Zhang et al., Phys. Rev. Lett. 67, 3452 (1991).

[12] I. I. Mazin et al., Phys. Rev. B 47, 538 (1993).

[13] V. P. Antropov et al., Phys. Rev. B 48, 7651 (1993).

[14] M. Capone, M. Fabrizio, C. Castellani, and E. Tosatti Rev. Mod. Phys. 81, 943 (2009).

[15] T. W. Ebbesen et al., Physica C 203, 163 (1992).

[16] B. Burk, Vincent H. Crespi, A. Zettl, and Marvin L. Cohen Phys. Rev. Lett. 72, 3706 (1994).

[17] T. Kosugi et al., J. Phys. Soc. Japan 78, 113704 (2009).

[18] A. Subedi and L. Boeri, arXiv:1103.4020

[19] We use the Quantum-espresso 20] code. K, C, and H atoms are described by ultrasoft pseudopotentials. The plane-wave (PW) cutoff is $60 \mathrm{Ry}$ for the wave-function, and 600 Ry for the charge. A $4 \times 4 \times 4$ electron-momentum grid and a Methfessel-Paxton smearing of 0.03 Ry are used in the electronic integration.

[20] P. Giannozzi et al., J. Phys.: Condens. Matter 21, 395502 (2009).

[21] Monoclinic, $a, b, c=8.707,5.912,12.97 \AA, \beta=92.77^{\circ}$

[22] Internal coordinates are reported in the additional materials [23].

[23] EPAPS Document No. XXX. For more information on EPAPS, see http://www.aip.org/pubservs/epaps.html.

[24] S. Baroni et al., Rev. Mod. Phys. 73, 515 (2001).

[25] P. B. Allen, Phys. Rev. B 6, 2577 (1972).

[26] M. Calandra et al., Phys. Rev. B 82, 165111 (2010).

[27] N. Marzari and D. Vanderbilt, Phys. Rev. B 56, 12847 (1997).

[28] A. A. Mostofi et al., Comput. Phys. Commun. 178, 685 (2008)

[29] F. Giustino et al., Phys. Rev. B 76, 165108 (2007)

[30] Wannierization has been performed on a $4 \times 4 \times 4$ electronmomentum mesh, by including the LUMO, LUMO+1, and $\mathrm{LUMO}+2$ states. Both the long-range hopping and inclusion of the first three LUMO's are needed to get localized orbitals and Wannierized bands in a perfect agreement with those computed in the PW (plane waves) basis set in a window of $0.7 \mathrm{eV}$ around the Fermi level.

[31] We use a $120 \times 120 \times 120$ electron-momentum grid, which guarantees convergence down to the temperature and smeating used in the present calculations $(T=150 \mathrm{~K}$ and $\sigma=4.3 \mathrm{meV}$, with $T=3 \sigma)$.

[32] W. L. McMillan, Phys. Rev. 167, 331 (1968).

[33] G. Giovannetti and M. Capone, Phys. Rev. B 83, 134508 (2011).

[34] C. Grimaldi et al., Phys. Rev. Lett. 75, 1158 (1995).

[35] M. Calandra and F. Mauri, Phys. Rev. Lett. 95, 237002 (2005).

[36] F. Ortmann, F. Bechstedt, and K. Hannewald, New J. Phys. 12, 023011 (2010). 


\title{
Supplemental material for the paper: Intercalant and intermolecular phonon assisted superconductivity in K-doped picene
}

\author{
Michele Casula, ${ }^{1}$ Matteo Calandra, ${ }^{1}$ Gianni Profeta ${ }^{2}$ and Francesco Mauri ${ }^{1}$ \\ 1 IMPMC, Université Paris 6, CNRS, 4 Place Jussieu, 7504 Paris, France \\ 2 SPIN-CNR - Dip. di Fisica Università degli Studi di L'Aquila, 67100 L'Aquila, Italy \\ Max-Planck Institute of Microstructure Physics, Weinberg 2, 06120 Halle, Germany
}

\section{GEOMETRY}

We report the coordinates of the 78 atoms in the monoclinic unit cell with $a, b, c=8.707,5.912,12.97 \AA, \beta=92.77^{\circ}$. In the Table, they are split into two groups, one for each $\mathrm{K}_{3}$-picene molecule, related each other by the $\mathrm{P} 2_{1}$ symmetry operations. The atomic symbols are in the first column. The coordinates are in crystal units.

\begin{tabular}{|c|c|c|c|c|c|c|}
\hline & \multicolumn{3}{|c|}{ molecule 1} & \multicolumn{3}{|c|}{ molecule 2} \\
\hline C & 0.4556221 & 0.6985366 & 0.5225742 & -0.4556221 & 0.1985366 & -0.5225742 \\
\hline C & 0.3666947 & 7916786 & 0.5978784 & -0.3666947 & 0.2916786 & -0.5978784 \\
\hline $\mathrm{C}$ & 0.4078295 & 0.6965432 & 0.4186143 & -0.4078295 & 0.1965432 & -0.4186143 \\
\hline $\mathrm{H}$ & 0.5603062 & 0.6067382 & 0.5464734 & -0.5603062 & 0.1067382 & -0.5464734 \\
\hline $\mathrm{C}$ & 0.2680166 & 0.7885757 & 0.3834603 & -0.2680166 & 0.2885757 & -0.3834603 \\
\hline $\mathrm{H}$ & 0.4802802 & 0.6105565 & 0.3645861 & -0.4802802 & 0.1105565 & -0.3645861 \\
\hline $\mathrm{C}$ & 0.1758863 & 0.9015619 & 0.4566338 & -0.1758863 & 0.4015619 & -0.4566338 \\
\hline $\mathrm{C}$ & 0.2192085 & 0.7895931 & 0.2754507 & -0.2192085 & 0.2895931 & -0.2754507 \\
\hline $\mathrm{C}$ & 0.0759228 & 0.8943093 & 0.2435217 & -0.0759228 & 0.3943093 & -0.2435217 \\
\hline $\mathrm{C}$ & 0.3100630 & 0.7014734 & 0.1996684 & -0.3100630 & 0.2014734 & -0.1996684 \\
\hline $\mathrm{C}$ & 0.2617866 & 0.6976639 & 0.0950588 & -0.2617866 & 0.1976639 & 50588 \\
\hline $\mathrm{H}$ & 0.4193296 & 0.6208 & 0.2225564 & 296 & 8887 & -0.2 \\
\hline $\mathrm{C}$ & 0.1210633 & 0.7886946 & 0.0646123 & -0.1210633 & 0.2886946 & -0.0646123 \\
\hline $\mathrm{H}$ & 0.3366770 & 0.6247067 & 0.0390565 & -0.3366770 & 0.1247067 & -0.0390565 \\
\hline $\mathrm{C}$ & 0.0298681 & 0.8877619 & 0.1371182 & -0.0298681 & 0.3877619 & -0.1371182 \\
\hline $\mathrm{H}$ & 0.0819831 & 0.7857442 & -0.0168198 & -0.0819831 & 0.2857442 & 0.0168198 \\
\hline $\mathrm{H}$ & -0.0771974 & 0.9716713 & 0.1101440 & 0.0771974 & 0.4716713 & -0.1101440 \\
\hline $\mathrm{C}$ & -0.0065371 & 1.0089684 & 0.3165922 & 0.0065371 & 0.5089684 & -0.3165922 \\
\hline $\mathrm{C}$ & 0.0469952 & 1.0175338 & 0.4200294 & -0.0469952 & 0.517 & -0.42 \\
\hline $\mathrm{H}$ & -0.1098166 & 1.1064455 & 0.2929669 & 0.1098166 & 0.6064455 & -0.2929669 \\
\hline $\mathrm{H}$ & -0.0159085 & 1.1243530 & 0.4725799 & 0.0159085 & 0.6243530 & -0.4725799 \\
\hline $\mathrm{C}$ & 0.2237032 & 0.8990497 & 0.5655413 & -0.2237032 & 0.3990497 & -0.56 \\
\hline $\mathrm{C}$ & 0.1396179 & 1.0059855 & 0.6385974 & -0.1396179 & 0.5059855 & -0.6385974 \\
\hline $\mathrm{C}$ & 0.1916193 & 1.0169922 & 0.7421052 & -0.1916193 & 0.5169922 & -0.7421052 \\
\hline $\mathrm{H}$ & 0.0315715 & 1.0922925 & 0.6160368 & -0.0315715 & 0.5922925 & -0.6160368 \\
\hline $\mathrm{C}$ & 0.3247277 & 0.9053904 & 0.7780469 & -0.3247277 & 0.4053905 & -0.7780469 \\
\hline $\mathrm{H}$ & 0.1258220 & 1.1133177 & 0.7972359 & -0.1258220 & 0.6133177 & -0.7972359 \\
\hline $\mathrm{C}$ & 0.4177289 & 0.7943654 & 0.7053137 & -0.4177289 & 0.2943654 & -0.7053137 \\
\hline $\mathrm{C}$ & 0.3729009 & 0.9021317 & 0.8836312 & -0.3729009 & 0.4021317 & -0.8836312 \\
\hline $\mathrm{C}$ & 0.5070607 & 0.7918688 & 0.9174399 & -0.5070607 & 0.2918688 & -0.9174399 \\
\hline $\mathrm{H}$ & 0.2970889 & 0.9733633 & 0.9403859 & -0.2970889 & 0.4733633 & -0.9403859 \\
\hline C & 0.6002138 & 0.6930755 & 0.8478521 & -0.6002138 & 0.1930755 & -0.8478521 \\
\hline $\mathrm{H}$ & 0.5342170 & 0.7751262 & 1.0001134 & -0.5342170 & 0.2751262 & -1.0001134 \\
\hline C & 0.5569571 & 0.6993506 & 0.7422534 & -0.5569571 & 0.1993506 & -0.7422534 \\
\hline $\mathrm{H}$ & 0.7001736 & 0.5946126 & 0.8756002 & -0.7001736 & 0.0946126 & -0.8756002 \\
\hline $\mathrm{H}$ & 0.6292360 & 0.6123495 & 0.6882365 & -0.6292360 & 0.1123495 & -0.6882365 \\
\hline K & 0.0968372 & 0.3605062 & 0.1955914 & -0.0968372 & -0.1394938 & -0.1955914 \\
\hline K & 0.3513565 & 0.3915392 & 0.8277846 & -0.3513565 & -0.1084608 & -0.8277846 \\
\hline $\mathrm{K}$ & 0.2382784 & 0.3669465 & 0.5169973 & -0.2382784 & -0.1330535 & -0.5169973 \\
\hline
\end{tabular}




\section{BROADENING AND q-DEPENDENCE OF $\lambda$}

In the Figure, we present the phonon momentum q-resolved $\lambda$ 's, evaluated as in Eq. 2 in the paper, as a function of the Gaussian smearing $\sigma$ of the delta functions. The plotted results are obtained by using a $120^{3}$ electron $\mathbf{k}$-point mesh. The calculations performed with $60^{3} \mathrm{k}$-point mesh give identical results down to $T=150 \mathrm{~K}$ and $\sigma=4.3 \mathrm{meV}$, with $T=3 \sigma$. The $\mathbf{q}$ vectors are reported in crystal units. The exact zero-smearing extrapolation leads to a strong q-dependence in $\lambda$, which can be seen only for $\sigma$ much smaller than the bandwidth $\Delta \epsilon(\ll 0.1 \mathrm{eV})$. In the inset, we show the q-integrated "adiabatic" and "non-adiabatic" $\lambda$. The "non-adiabatic" $\lambda$ is extrapolated with respect to both $\sigma$ and $T$.

Note the divergence of $\lambda_{\mathbf{q}}^{A D}$ at the zone center. This occurs in general in presence of optical modes, because of the divergence of the nesting factor $J_{\mathbf{q}}$ at $\mathbf{q}=0$. For this reason, in the "adiabatic" $\mathbf{q}$-averaged $\lambda^{A D}$ the $\Gamma$ point has been excluded. In the "non-adiabatic" case, $\lambda_{\mathbf{q}}$ is well behaved also at $\Gamma$.

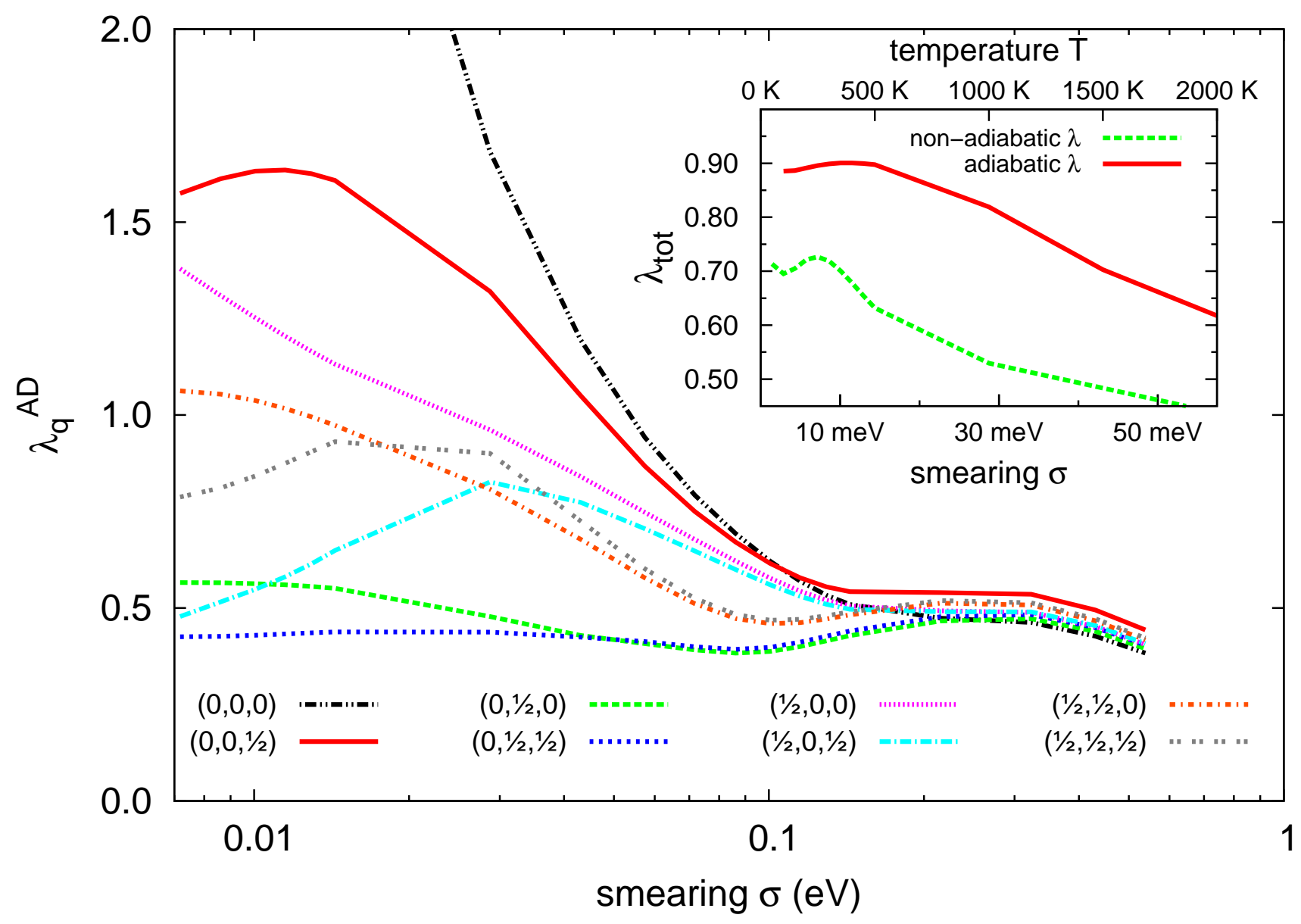

COUPLING STRENGTH AVERAGE

From $\lambda_{\mathbf{q}}^{A D}$, we can quantify the $\mathbf{q}$-dependent coupling strength average per molecule, by the formula $2 \lambda_{\mathbf{q}}^{A D} N(0) / J_{\mathbf{q}}$, where $J_{\mathbf{q}}=\sum_{\mathbf{k}, n, m} \delta\left(\epsilon_{\mathbf{k}, n}\right) \delta\left(\epsilon_{\mathbf{k}+\mathbf{q}, m}\right) / N_{k}$ is the nesting factor, $\lambda_{\mathbf{q}}^{A D}$ is summed over the phonon modes, and the factor of 2 accounts for the number of molecules in the unit cell. One obtains the values (in meV) $\{183,193,131,133,239$, $146,191,161\}$ for the q-vectors (in crystal units) $\left\{(0,0,0),\left(0,0, \frac{1}{2}\right),\left(0, \frac{1}{2}, 0\right),\left(0, \frac{1}{2}, \frac{1}{2}\right),\left(\frac{1}{2}, 0,0\right),\left(\frac{1}{2}, 0, \frac{1}{2}\right),\left(\frac{1}{2}, \frac{1}{2}, 0\right)\right.$, $\left.\left(\frac{1}{2}, \frac{1}{2}, \frac{1}{2}\right)\right\}$, respectively. Note that even at $\Gamma$ the ratio is well defined, as the divergences of both $\lambda_{\Gamma}^{A D}$ and $J_{\Gamma}$ cancel out. 